\title{
Nonequilibrium Linear Response for Markov Dynamics, II: Inertial Dynamics
}

\author{
Marco Baiesi ${ }^{1,2}$, Eliran Boksenbojm² ${ }^{2}$ Christian Maes $^{2}$, Bram Wynants ${ }^{2, *}$ \\ 1: Dipartimento di Fisica, Università di Padova, Via Marzolo 8, 35131 Padova, Italy \\ 2: Instituut voor Theoretische Fysica, K. U. Leuven, B-3001 Leuven, Belgium \\ *bram.wynants@fys.kuleuven. be
}

December 3, 2009

\begin{abstract}
We continue our study of the linear response of a nonequilibrium system. This Part II concentrates on models of open and driven inertial dynamics but the structure and the interpretation of the result remain unchanged: the response can be expressed as a sum of two temporal correlations in the unperturbed system, one entropic, the other frenetic. The decomposition arises from the (anti)symmetry under time-reversal on the level of the nonequilibrium action. The response formula involves a statistical averaging over explicitly known observables but, in contrast with the equilibrium situation, they depend on the model dynamics in terms of an excess in dynamical activity. As an example, the Einstein relation between mobility and diffusion constant is modified by a correlation term between the position and the momentum of the particle.
\end{abstract}

\section{Introduction}

The linear response of equilibrium systems to a time-dependent shift in the energy has been extensively studied throughout the twentieth century. It is an important question in the construction of nonequilibrium statistical mechanics to find suitable extensions, most interestingly with a systematic physical interpretation. Many efforts have been devoted to such a programme [3, 5, 7, 9, 13, 16, 17, 23, 24, 25, 26, 27, 28, and the present paper is contributing a second part to what we believe is a unifying and yet novel approach to the subject. In the previous Part I [2] we have shown how to think of this extension for Markov jump processes and for overdamped diffusions. In the present continuation we deal with more general processes including underdamped diffusions. Their state space contains both position and momentum degrees of freedom and hence there are important changes with respect to the analysis in Part I, mostly having to do with the nature of time-reversal and dissipation. For inertial systems the time-reversal includes changing the sign of the momenta, which are the variables connected with energy dissipation, as this is power lost by the frictional forces exerted on momenta. More generally, the Hamiltonian structure adds some nontrivial aspects to the analysis. Nevertheless the strategy with its statistical interpretation holds basically unchanged.

The response formula gives the generalized susceptibilities for changes in the potential $U \rightarrow U-h_{s} V$ depending on time $s$ in a quantity $Q$ at time $t \geq s$ in the form [1]

$$
\langle Q(t)\rangle^{h}-\langle Q(t)\rangle=\frac{1}{2}\langle\mathcal{S}(\omega) Q(t)\rangle-\frac{1}{2}\langle\tau(\omega) Q(t)\rangle
$$

where $\omega$ is the history, $\mathcal{S}(\omega)$ is the path-dependent excess in entropy flux and $\tau(\omega)$ is the path-dependent excess in dynamical activity to linear order in $h_{s}$, called frenesy [2]. Excess refers to the perturbation 
$V$. The brackets $\langle\ldots\rangle$ and $\langle\ldots\rangle^{h}$ denote expectations in the unperturbed and perturbed process, respectively. The structure is thus similar to that in equilibrium, with responses written in term of correlations estimated in the unperturbed regime. Before giving the general explanations, we start with an example, an inertial Brownian motion under nonequilibrium driving.

We consider a particle with momentum and position $(q, p) \in \mathbb{R}^{2 d}$ undergoing

$$
\begin{gathered}
\mathrm{d} q=p \mathrm{~d} t \\
\mathrm{~d} p=-\nabla U(q) \mathrm{d} t+F(q) \mathrm{d} t-\gamma p \mathrm{~d} t+h_{t} E \mathrm{~d} t+\sqrt{2 \gamma / \beta} \mathrm{d} B(t)
\end{gathered}
$$

where $\gamma$ is the friction by the heat bath at inverse temperature $\beta$ and $\mathrm{d} B(t)$ is a standard white noise. With a sufficiently confining potential $U$, a stationary distribution $\rho$ settles for a nonequilibrium due to the presence of a non-gradient force $F$. The perturbation is in the form of a constant external field $E$ with amplitude $h_{t}$. We look at the excesses, first in entropy flux: for a path $\omega$ during $[0, t]$,

$$
\mathcal{S}(\omega)=\beta \int_{0}^{t} \mathrm{~d} s E \cdot p(s) h_{s}
$$

is the excess entropy flux by the field $E$ (heat transmitted to the reservoir divided by the temperature, taking Boltzmann's constant equal to one). Secondly we must identify the path-dependent frenesy, that is, the linear order (in $h$ ) in the excess of dynamical activity or generalized escape rate. We give it here without further explanation to return to it in Sections 2 and 3 . For the present example it is given by

$$
\tau(\omega)=-\frac{\beta}{\gamma} \int_{0}^{t} h_{s}[\mathrm{~d} s(\nabla U-F)+\mathrm{d} p(s)] \cdot E
$$

where in the square brackets and from (21) one discovers the thermostating forces for the original dynamics, friction plus forcing from the heat bath:

$$
(\nabla U-F) \mathrm{d} s+\mathrm{d} p(s)=-\gamma p \mathrm{~d} s+\sqrt{2 \gamma / \beta} \mathrm{d} B(s)
$$

Observe that $\tau$ is symmetric under time-reversing the paths (together with flipping the momentum). If for example we take the momentum $p(t)$ as the observable for which we seek the linear response, the result is like (1):

$$
\langle p(t)\rangle^{h}-\langle p(t)\rangle=\frac{1}{2}\langle\mathcal{S} p(t)\rangle-\frac{1}{2}\langle\tau p(t)\rangle
$$

or, in differential form, yielding the nonequilibrium dynamic mobility with $0<s<t$,

$$
\begin{aligned}
\left.\frac{\delta}{\delta h_{s}}\langle p(t)\rangle_{\rho}^{h}\right|_{h=0}= & \frac{\beta}{2}\langle E \cdot p(s) p(t)\rangle_{\rho} \\
& +\frac{\beta}{2 \gamma}\langle E \cdot[\nabla U(q(s))-F(q(s))] p(t)\rangle_{\rho}+\frac{\beta}{2 \gamma} \frac{\mathrm{d}}{\mathrm{d} s}\langle E \cdot p(s) p(t)\rangle_{\rho}
\end{aligned}
$$

We have added the subscript $\rho$ to indicate that we are looking here at the stationary situation, although this is just a standard choice and is not necessary for our approach. In equilibrium, when $F$ would itself be a gradient, one has time-reversal invariance and one easily checks that the second line in (515) contributes exactly the same as in the first line, obtaining the standard relation between mobility (left-hand side) and diffusion $\beta\langle E \cdot p(s) p(t)\rangle^{\mathrm{eq}}$ in terms of momentum correlations. This example, the nonequilibrium relation between mobility and diffusivity, has been visited already by various groups including [11, 12, 4, 29] containing various and different useful response relations. We will revisit it as Example 5.2.

The purpose of the present paper is to explain the general algorithm for identifying the terms in (11) resulting e.g. in (5) and to add the statistical dynamical meaning. In the next section we explain 
the general strategy on the level of path-space distributions. Section 3 fills in the details for inertial diffusions as in the example above. In Section 4 we connect our formulation with previous ones, in particular those obtained via first order perturbation theory. Finally there are more examples and comparisons in Section [5, including a generalized Einstein relation (27).

\section{General strategy to linear response}

We start with an algorithm for arriving at the general linear response formula (11), outlined in [1]. We suppose variables $(q, p) \in \mathbb{R}^{2 d}$ which trace out some path or trajectory during a time-interval $[0, t]$. We can think of a Hamiltonian flow to which we have added nonequilibrium forces by various contacts with the environment. At the initial time $s=0$ the system is found in a nonequilibrium regime, possibly a steady state. We are interested in some observation at time $t$ when we perturb the Hamiltonian by adding $-h_{s} V$, with $t>s>0$. Let us also add that what follows is greatly independent of the details of the dynamics.

We compare the perturbed system with the original one yielding a probability density $\mathcal{P}^{h}(\omega)$ for paths $\omega=(q(s), p(s))$ for times $s \in[0, t]$. We assume that this probability density can be written in terms of an action $\mathcal{A}$, simply as

$$
\mathcal{P}^{h}(\omega) \equiv \frac{\operatorname{Prob}^{h}[\omega]}{\operatorname{Prob}[\omega]}=e^{-\mathcal{A}(\omega)}
$$

That amounts to supposing $\operatorname{Prob}[\omega]=0 \Rightarrow \operatorname{Prob}^{h}[\omega]=0$. Thus $\mathcal{P}^{h}(\omega)$ gives the changed plausibility of trajectories $\omega$ on the considered scale of reduced description. In the case of Markov dynamics, $\mathcal{A}=\int_{0}^{t} \mathrm{~d} s L_{s}(q(s), d q(s) ; p(s), d p(s))$ is a time-integral. A natural decomposition of $\mathcal{A}$ can be made with the time-reversal $\theta$ which changes $\omega$ into $\theta \omega$ with $(\theta \omega)_{s}=(q(t-s),-p(t-s))$. Then, $\mathcal{A}=(\mathcal{T}-\mathcal{S}) / 2$ with

$$
\begin{aligned}
\mathcal{S} & =\mathcal{A} \theta-\mathcal{A}, \\
\mathcal{T} & =\mathcal{A} \theta+\mathcal{A}, \\
\mathcal{P}^{h}(\omega) & =e^{-\mathcal{T}(\omega) / 2} e^{\mathcal{S}(\omega) / 2}
\end{aligned}
$$

The source-term of time-symmetry breaking is $\mathcal{S}$ and, under typical nonequilibrium conditions, it is exactly the excess in physical entropy flux due to the perturbation. For Markov dynamics that is being implemented by the condition of local detailed balance, but it can be more generally derived from mechanical beginnings, see [20, 30] for derivations starting from a Hamiltonian evolution. For constructing Markov models the condition of local detailed balance was emphasized in [14, 10] and it underlies what is called the fluctuation symmetry for the variable entropy production [18, 19, 15. For the general algorithm, if we know the excess entropy flux we know $\mathcal{S}$, see (3) as a first example.

The time-symmetric term $\mathcal{T}$ is an excess in the time-integrated instantaneous dynamical activity. It was discussed in [22, 21] for overdamped diffusions and for Markov jump processes respectively. By looking at (6) we see that, of two trajectories having the same entropy flux, the one with the lowest excess in activity is most probable. Formally, the dynamical activity in $(q, p)$ is the rate of escape from that phase space point. We will see it implemented in Section 3 for underdamped diffusions. In the first example (4) and as already mentioned there,

$$
\mathcal{T}(\omega)=-\int_{0}^{t} \mathrm{~d} s h_{s} \frac{\partial V}{\partial q}(q(s)) \mathcal{F}(s)+O\left(h^{2}\right)
$$

for random forcing $\mathcal{F}(s)=\frac{1}{D}[-\gamma p(s)+\sqrt{2 D} \mathrm{~d} B(s) / \mathrm{d} s]$, with $D=\gamma / \beta$.

Clearly then, the response can be calculated from

$$
\langle Q(t)\rangle^{h}=\left\langle Q(t) e^{\mathcal{S} / 2-\mathcal{T} / 2}\right\rangle
$$


where the right-hand side averages over the unperturbed process. The created entropy flux is dissipating the extra energy in the environment which is itself of order $h$. Therefore, the excess entropy $\mathcal{S}$ is already linear in $h$. The dynamical activity can be linearized to $\mathcal{T}=\tau+O\left(h^{2}\right)$ and we obtain (1):

$$
\langle Q(t)\rangle^{h}=\langle Q(t)\rangle+\frac{1}{2}\langle\mathcal{S} Q(t)\rangle-\frac{1}{2}\langle\tau Q(t)\rangle
$$

which is the same structure of linear response as in [1, 2].

We can in fact also consider $t=0$ in (7) from which we see that

$$
\langle\mathcal{S} Q(0)\rangle=\langle\tau Q(0)\rangle
$$

Since in equilibrium there is full time-reversal invariance, we know for the right-hand side of (8) that $\langle\tau Q(t)\rangle^{\text {eq }}=\langle\tau \epsilon Q(0)\rangle^{\text {eq }}$, due to time-reversal invariance of $\tau\left(\epsilon=\epsilon_{Q}\right.$ is the sign of $Q$ upon time-reversal). Similarly $\langle\mathcal{S} Q(t)\rangle^{\mathrm{eq}}=-\langle\mathcal{S} \epsilon Q(0)\rangle^{\mathrm{eq}}$ because $\mathcal{S}$ is antisymmetric. Hence, by (8),

$$
-\langle\tau Q(t)\rangle^{\mathrm{eq}}=\langle\mathcal{S} Q(t)\rangle^{\mathrm{eq}}
$$

and we recover from (7) the fluctuation-dissipation relation,

$$
\langle Q(t)\rangle^{h}-\langle Q(t)\rangle^{\mathrm{eq}}=\langle\mathcal{S} Q(t)\rangle^{\mathrm{eq}}
$$

for equilibrium, with the standard interpretation that the linear response of a quantity to a perturbation is given by its correlation with the entropy that the perturbation produces. Out of equilibrium, the second term originating from the dynamical activity $\tau$, what we like to call the frenetic term, is not reducible to the (first) entropic term except for the relation (8). Implementing it in (7), we can still write that for all times $t \geq 0$

$$
\langle Q(t)\rangle^{h}-\langle Q(t)\rangle=\left\langle\mathcal{S} \frac{Q(t)-\epsilon Q(0)}{2}\right\rangle-\left\langle\tau \frac{Q(t)-\epsilon Q(0)}{2}\right\rangle
$$

where the excesses $\mathcal{S}$ (in entropy flux) and $\tau$ (in activity) are over the time-interval $[0, t]$, linear in the amplitudes $h_{s}$. The second term in the right-hand side of (9) vanishes in equilibrium.

\section{Markov dynamics for underdamped diffusions}

The generic model that adds a specific dynamics to the previous section has states $(q, p)=\left(q_{1}, q_{2}, \ldots, q_{n}\right.$; $\left.p_{1}, p_{2}, \ldots, p_{n}\right) \in \mathbb{R}^{2 n}$ of positions and momenta. We imagine a weak coupling with equilibrium reservoirs. That is: at each $1 \leq i \leq n$ are attached a standard white noise $\mathrm{d} B_{i}(t)$ with diffusion constant $D$ and a friction coefficient $\gamma_{i}$ to model a heat bath at temperature $D / \gamma_{i}=T_{i}$ :

$$
\begin{aligned}
\mathrm{d} q_{i} & =p_{i} \mathrm{~d} t \\
\mathrm{~d} p_{i} & =F_{i}(q)-\frac{\partial U}{\partial q_{i}} \mathrm{~d} t-\gamma_{i} p_{i} \mathrm{~d} t+h_{t} \frac{\partial V}{\partial q_{i}} \mathrm{~d} t+\sqrt{2 D} \mathrm{~d} B_{i}(t)
\end{aligned}
$$

for a given potential $U(q)$ taking care of the coupling and pinning of the positions. The pinning is also thought to confine the positions to some finite volume. Besides the possibility of having multiple temperatures $T_{i}$ to drive the system away from equilibrium, we also add a nonconservative forcing $F_{i}(q)$. Finally, the perturbation is $V(q)$ and thus adds a potential to the unperturbed Hamiltonian $H_{o}=\sum_{i} p_{i}^{2} / 2+U(q) \rightarrow H_{o}-h_{s} V(q)$ with small time-dependent amplitude $h_{s}$ for $s \geq 0$.

Alternatively, we can write the Fokker-Planck equation for the unperturbed system as

$$
\frac{\mathrm{d}}{\mathrm{d} t} \mu_{t}+\nabla \cdot J_{\mu_{t}}=0
$$


for $\nabla=\left(\nabla_{q}, \nabla_{p}\right)$ and for current $J_{\mu}=\left(J_{\mu}^{q}, J_{\mu}^{p}\right)$ with

$$
J_{\mu}^{q}=p \mu, \quad J_{\mu}^{p}=\left(F-\nabla_{q} U\right) \mu-\gamma p \mu-D \nabla_{p} \mu
$$

to be understood with diagonal matrices $\gamma$ and $D$. The backward generator $L$ is

$$
L f=p \nabla_{q} f+\left(F-\nabla_{q} U-\gamma p\right) \nabla_{p} f+\nabla_{p} D \nabla_{p} f
$$

Let us now apply the algorithm. In a time-interval $[0, t]$ the time-integrated entropy flux in excess (by the perturbation) is made from the extra heat into the various reservoirs:

$$
\mathcal{S}=\sum_{i=1}^{n} \frac{1}{T_{i}} \int_{0}^{t} h_{s} \frac{\partial V}{\partial q_{i}}(q(s)) p_{i}(s) \mathrm{d} s
$$

On the other hand, the excess in dynamical activity is obtained by checking the excess action $\mathcal{A}$ of the previous section and linearizing the time-symmetric part:

$$
\tau(\omega)=\frac{1}{D} \sum_{i} \int_{0}^{t} h_{s} \frac{\partial V}{\partial q_{i}}(q(s))\left[\left\{F_{i}(q(s))-\frac{\partial U}{\partial q_{i}}(q(s))\right\} \mathrm{d} s-\mathrm{d} p_{i}(s)\right]
$$

The last stochastic integral (there is no difference here between the Itô and the Stratonovich conventions) can still be worked out when inserting $\tau$ in the correlation function (second term in (1)). We get

$$
\begin{aligned}
& \int_{0}^{t} \mathrm{~d} s h_{s}\left\langle\frac{\partial V}{\partial q_{i}}(q(s)) \mathrm{d} p_{i}(s) Q(t)\right\rangle_{\mu}= \\
& \int_{0}^{t} h_{s} \frac{\mathrm{d}}{\mathrm{d} s}\left\langle\frac{\partial V}{\partial q_{i}}(q(s)) p_{i}(s) Q(t)\right\rangle_{\mu} \mathrm{d} s-\sum_{j} \int_{0}^{t} h_{s}\left\langle\frac{\partial^{2} V}{\partial q_{j} \partial q_{i}}(q(s)) p_{j}(s) p_{i}(s) Q(t)\right\rangle_{\mu} \mathrm{d} s
\end{aligned}
$$

for an arbitrary starting density $\mu(q, p)$. As a consequence the total linear response function for the underdamped diffusion model (10) is

$$
\left.\frac{\delta}{\delta h_{s}}\langle Q(t)\rangle_{\mu}^{h}\right|_{h=0}=\sum_{i=1}^{n} \frac{1}{2 T_{i}}\left\langle\frac{\partial V}{\partial q_{i}}(q(s)) p_{i}(s) Q(t)\right\rangle_{\mu}-\frac{1}{2}\langle\tau(s) Q(t)\rangle_{\mu}
$$

with

$$
\begin{aligned}
& D\langle\tau(s) Q(t)\rangle_{\mu}=\sum_{i}\left\langle\frac{\partial V}{\partial q_{i}}(q(s))\left[F(q(s))-\frac{\partial U}{\partial q_{i}}(q(s))\right] Q(t)\right\rangle_{\mu} \\
& -\frac{\mathrm{d}}{\mathrm{d} s} \sum_{i}\left\langle\frac{\partial V}{\partial q_{i}}(q(s)) p_{i}(s) Q(t)\right\rangle_{\mu}+\sum_{i, j}\left\langle\frac{\partial^{2} V}{\partial q_{j} \partial q_{i}}(q(s)) p_{j}(s) p_{i}(s) Q(t)\right\rangle_{\mu}
\end{aligned}
$$

Note that all the observables (within the expectations) are explicitly known. The formula remains valid whether $\mu=\rho$ (stationary regime) or otherwise (transient regime). Some specific examples follow in Section 5 .

\section{From the Dyson expansion}

Linear response is first order perturbation theory and here we assume smooth behavior. We could therefore also set up a simple Taylor expansion, or call it time-dependent perturbation theory, as for example in [13, 24], also for nonequilibria. Below we sketch the immediate problem of explicitness, but 
true enough, the problem is not so much finding a linear response formula but rather to get a useful form that also yields interesting relations with physical concepts within fluctuation theory.

We prepare the system at time $t=0$ according to its stationary distribution $\rho$; the perturbation $-h V$ is added for positive times - we think of the set-up in (10). Therefore, for times $t \geq 0$ the dynamics has (backward) generator (working on observables)

$$
L^{h}=L+h \nabla_{q} V \cdot \nabla_{p}, \quad L=(F-\nabla U-\gamma p) \cdot \nabla_{p}+p \cdot \nabla_{q}+\nabla_{p} D \nabla_{p}
$$

with $\gamma$ written as diagonal matrix. For the change in expectations at times $t$ with respect to what we had at time zero

$$
\langle Q(t)\rangle^{h}-\langle Q(t)\rangle=\int \mathrm{d} p \mathrm{~d} q \rho(q, p)\left(e^{t L^{h}}-e^{t L}\right) Q(q, p)
$$

we get the linear order

$$
e^{t L^{h}}-e^{t L}=\int_{0}^{t} e^{(t-s) L}\left(L^{h}-L\right) e^{s L} \mathrm{~d} s+O\left(h^{2}\right)
$$

Or, always to leading order in $h \downarrow 0$,

$$
\frac{1}{h}\left[\langle Q(t)\rangle^{h}-\langle Q(t)\rangle\right]=\int_{0}^{t} \mathrm{~d} s R_{Q V}(t, s)
$$

with response function

$$
R_{Q V}(t, s)=\int \mathrm{d} q \mathrm{~d} p \rho(q, p) \nabla_{q} V \cdot \nabla_{p} e^{(t-s) L} Q(q, p)
$$

(The dependence on time $s$ and on time $t$ is made for greater generality in case the perturbation is time-dependent through $h_{s}$.) Equation (18) is directly useful when $\rho$ is given, as in equilibrium, but not otherwise. With partial integration we obtain

$$
\begin{aligned}
R_{Q V}(t, s) & =-\left\langle\frac{\nabla_{p} \rho}{\rho}(q(s), p(s)) \cdot \nabla_{q} V(s) Q(t)\right\rangle_{\rho} \\
& =\beta \frac{\mathrm{d}}{\mathrm{d} s}\langle V(s) Q(t)\rangle-\beta\left\langle\left[p(s)+\frac{\nabla_{p} \rho}{\beta \rho}(q(s), p(s)) \cdot \nabla_{q} V(q(s)) Q(t)\right\rangle_{\rho}\right.
\end{aligned}
$$

where in the second line we have introduced the equilibrium form (first term) at inverse temperature $\beta$ as a reference. The term $\nabla_{p} \rho / \rho=\nabla_{p} \ln \rho$ is unknown in general which brings the lack of explicitness.

We can still make the connection with [6]. We define a new current $\mathcal{J}_{\rho}$ (with respect to $J_{\rho}$ of (12) that remains divergenceless:

$$
\mathcal{J}_{\rho}=\left(J_{\rho}^{q}+\nabla_{p} \rho / \beta, J_{\rho}^{p}-\nabla_{q} \rho / \beta\right), \quad \nabla \cdot \mathcal{J}_{\rho}=\nabla \cdot J_{\rho}=0
$$

so that it still satisfies the same Fokker-Planck equation (11) (stationary for $\mu_{t}=\rho$ ). In terms of this new current we get

$$
R_{Q V}(t, s)=\beta \frac{\mathrm{d}}{\mathrm{d} s}\langle V(s) Q(t)\rangle_{\rho}-\beta\left\langle\frac{\mathcal{J}_{\rho}}{\rho}(s) \cdot \nabla_{q} V(s) Q(t)\right\rangle_{\rho}
$$

which is exactly the formula for the response relation in [6]. Indeed, as also pointed out in [29], the nonequilibrium fluctuation-response relation reduces to the equilibrium fluctuation-dissipation relation when viewed from a "Lagrangian frame," moving with the local velocity of the system.

Let us now see how the above expressions are mathematically related to our formula (11). As above and as in [6] we restrict ourselves to having one environment temperature $\beta^{-1}$. 
The crucial term to rewrite in (16) and (17) is the one with the derivative with respect to time. By definition of the backward generator $L$, such terms can be rewritten using

$$
\frac{\mathrm{d}}{\mathrm{d} s}\left\langle\frac{\partial V}{\partial q_{i}}(q(s)) p_{i}(s) Q(t)\right\rangle_{\rho}=\frac{\mathrm{d}}{\mathrm{d} s}\left\langle\frac{\partial V}{\partial q_{i}}(q(0)) p_{i}(0) Q(t-s)\right\rangle_{\rho}=-\left\langle\frac{\partial V}{\partial q_{i}}(q(s)) p_{i}(s) L Q(t)\right\rangle_{\rho}
$$

where $\rho$ is again the stationary measure. Again, this can be rewritten using the definition of the adjoint generator $L^{*}$ : for smooth functions $f, g$,

$$
\int \mathrm{d} p \mathrm{~d} q \rho(q, p) f(q, p) L g(q, p)=\int \mathrm{d} p \mathrm{~d} q \rho(q, p) g(q, p) L^{*} f(q, p)
$$

or,

$$
L^{*}=-p \nabla_{q}-\left(F-\nabla_{q} U-\gamma p\right) \nabla_{p}+\nabla_{p} D \nabla_{p}+2 \frac{\nabla_{p} \rho}{\rho} \cdot \nabla_{p}
$$

Clearly $L^{*}$ directly depends on the stationary measure. Its definition also implies that

$$
\left\langle\frac{\partial V}{\partial q_{i}}(q(s)) p_{i}(s) L Q(t)\right\rangle_{\rho}=\left\langle L^{*}\left(\frac{\partial V}{\partial q_{i}} p_{i}\right)(q(s), p(s)) Q(t)\right\rangle_{\rho}
$$

If we now use the explicit form of $L^{*}$ in this, and plug the result back in (16), then we indeed get (19).

The derivation above is generalizable to the transient case, in which the response function gets written as

$$
\begin{aligned}
R_{Q V}(t, s) & =-\left\langle\frac{\nabla_{p} \mu_{s}}{\mu_{s}}(q(s), p(s)) \cdot \nabla_{q} V(s) Q(t)\right\rangle_{\mu} \\
& =\beta \frac{\mathrm{d}}{\mathrm{d} s}\langle V(s) Q(t)\rangle_{\mu}-\beta\left\langle\frac{\mathcal{J}_{\mu_{s}}}{\mu_{s}}(s) \cdot \nabla_{q} V(s) Q(t)\right\rangle_{\mu}
\end{aligned}
$$

\section{$5 \quad$ Examples}

In order to illustrate the algorithm in concrete terms, we collect here a number of physically interesting examples of nonequilibrium systems following the Langevin equations (10): we remind that a potential $U$ determines conservative forces $\left(\partial U / \partial q_{i}\right)$, but the degrees of freedom $\left(q_{i}, p_{i}\right)$ can be at different temperatures $T_{i}$, generating a regime out of equilibrium. For simplicity we have fixed a diffusion constant $D$. The respective reservoirs are at equilibrium and they impose the Einstein relation with the friction coefficients $\gamma_{i}=T_{i} / D$. Another way of going out of equilibrium is to introduce nonconservative forces $\left(F_{i}\right)$, such as external fields that are rotational. A final possibility is to start from an initial condition that is not stationary, and thus to observe the response in a transient regime. Numerical results are better presented with integrated responses, for example by considering the generalized susceptibility

$$
\chi(t)=\int_{0}^{t} \mathrm{~d} s R_{Q V}(t, s)=\frac{\langle Q(t)\rangle^{h}-\langle Q(t)\rangle}{h}
$$

for small constant $h_{s}=h$ for all times $s>0$.

\subsection{Langevin particle in a periodic potential}

Recently there have been experiments testing the response of an overdamped particle (high viscosity limit) in a periodic potential [8]. In the previous paper [2] we have discussed simulations of that system. Here we look for the changes in an underdamped set-up, allowing e.g. for the particle to have a considerable inertia and to obey a noisy Hamiltonian dynamics. 
We denote the position by $q(t) \in S^{1}$ (on the circle) and the momentum by $p(t) \in \mathbb{R}$ and we choose for simplicity a particle with mass equal to one. Then, the equations (10) simplify to

$$
\begin{aligned}
& \mathrm{d} q(t)=p(t) \mathrm{d} t \\
& \mathrm{~d} p(t)=a(t) \mathrm{d} t-\gamma p(t) \mathrm{d} t-h_{t} g(t) \mathrm{d} t+\sqrt{2 D} \mathrm{~d} B(t)
\end{aligned}
$$

where we abbreviate

$$
\begin{array}{ll}
a(t)=f-\frac{\mathrm{d} U}{\mathrm{~d} q}(q(t)) & \quad \text { (deterministic force) } \\
g(t)=-\frac{\mathrm{d} V}{\mathrm{~d} q}(q(t)) & \quad \text { (perturbing force) }
\end{array}
$$

The nonconservative force $f$ is the driving and is taken constant over the circle, thus effectively tilting the conservative potential. At time $s=0$ the unperturbed system is in the stationary nonequilibrium $\rho$ corresponding to (23). Hence, the integrated correlations are

$$
\begin{array}{rlr}
C(t) & =\frac{1}{h}\langle\mathcal{S}(\omega) Q(t)\rangle \quad \quad \text { (entropic term) } \\
& =-\beta \int_{0}^{t} \mathrm{~d} s\langle p(s) g(s) Q(t)\rangle_{\rho} \\
K(t) & =-\frac{1}{h}\langle\tau(\omega) Q(t)\rangle \quad \text { (minus frenetic term) } \\
& =\frac{1}{D}\left\{\int_{0}^{t} d s\langle a(s) g(s) Q(t)\rangle_{\rho}-\int_{0}^{t}\langle\mathrm{~d} p(s) g(s) Q(t)\rangle_{\rho}\right\} \\
C_{\mathrm{ne}}(t) & =\frac{C(t)+K(t)}{2}
\end{array}
$$

Contrary to Part I, here we embed $\beta$ 's in the definitions of correlation functions: the integrated response relation is thus $\chi(t)=C_{\text {ne }}(t)$. We take $V(q)=U(q)=\cos q$ like in previous works [8, 2], and again also $Q=U$. Since the perturbation $g$ is the gradient of a potential $V$,

$$
C^{*}(t)=\beta\left[\langle V(t) Q(t)\rangle_{\rho}-\langle V(0) Q(t)\rangle_{\rho}\right]
$$

is an alternative to $C(t)$ for expressing the entropic term (from time-integrating the excess in entropy flux).

In Fig. 1 we visualize the various terms (see caption of the figure for more details), for three scenarios with different viscosity, increasing from left to right. The response is well reproduced by $C_{\mathrm{ne}}(t)$ of (26) even if we perform a numerical integration with $d t=10^{-3}$. Oscillations in the response are visible for small viscosity; at higher friction there is a monotonous drift toward a new stationary state (right panel in Fig. 11).

In Fig. 2 we can follow the response as a function of the driving $f$, with $f=0$ for equilibrium. For $f \neq 0$ the entropic term $C(t)$ can be quite different from $C_{\text {ne }}(t)$.

\subsection{A generalized Einstein relation}

We try to imagine here a collection of independent particles with no external potential $(U=0)$ except for keeping the particles in a large cylinder filled with some fluid. The motion is inertial and driven, e.g. with a driving $(F(q))$ resulting from steady differential rotation of the fluid. We consider therefore the general Markov dynamics (10), but the equation now refers to a single particle with $j, k$ being possibly different space directions. We are interested in the (long) transient regime in which the position diffuses. 


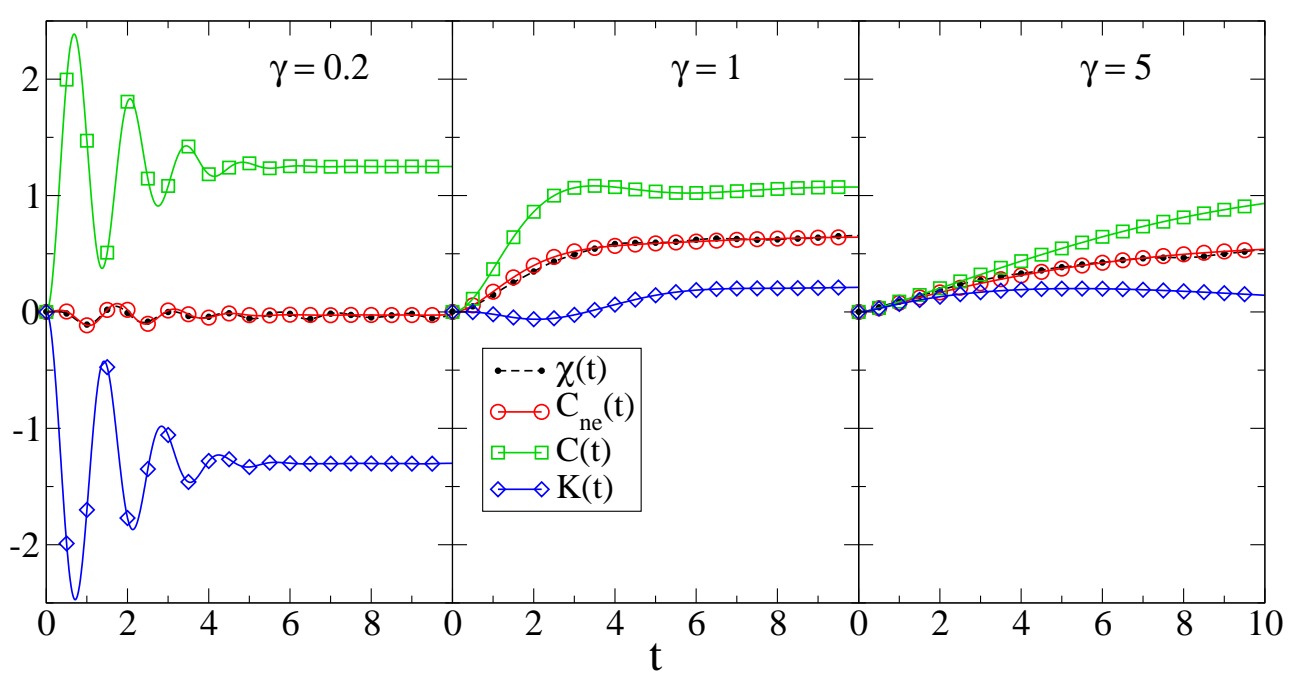

Figure 1: Integrated correlation functions of the excess in entropy production $C(t)$, of the frenesy $K(t)$, and their average $C_{\text {ne }}(t)$ giving the response in nonequilibrium, and the integrated response $\chi(t)$ calculated directly with $h=0.01$. Panels are for simulations with various friction coefficients: $\gamma=0.2$ (left), $\gamma=1$ (center), and $\gamma=5$ (right). Other parameters: $T=1 / \beta=0.4, f=0.9$. The inertial regime is less sensitive to the perturbation, as $\chi(t)$ displays only a small wiggling: a high entropy production is almost compensated by a high activity. For larger friction we recover previously studied overdamped scenarios; with this setting $(f \lesssim 1)$ the frenesy is close to zero compared with the excess entropy, and their combination yields $C_{\mathrm{ne}} \approx C / 2$.

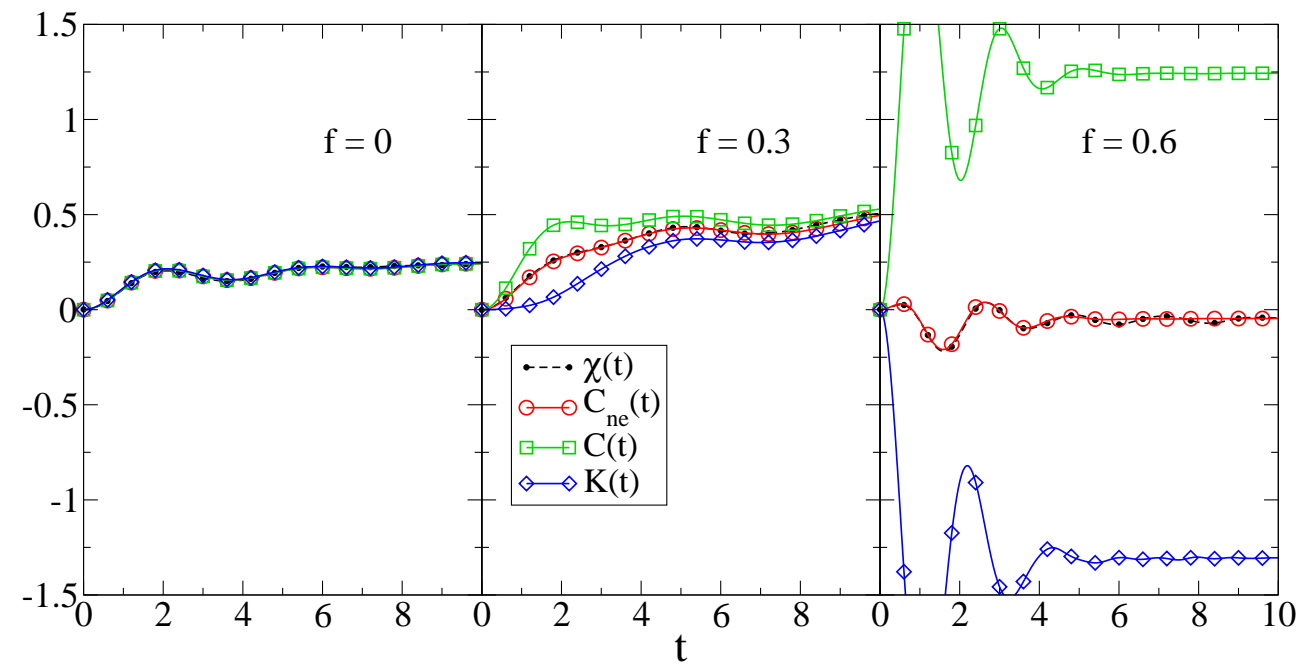

Figure 2: As in figure 1, but with fixed $\gamma=0.2$ and varying force $f$, from left to right: $f=0$ (equilibrium), $f=0.3$, and $f=0.6$ (case $f=0.9$ is in the previous figure). In equilibrium all the terms coincide, as expected.

Initially the particle is found at $(q(0), p(0))$ from a density $\mu$ on phase space.

As perturbation we take $V(q)=q_{j}$ for a fixed direction $j$, and the field $h_{t}=h$ is supposed constant in time. To observe is the variable $Q=p_{k}$ for possibly another direction $k$. Again, in this case the response function is related to the mobility of the system, i.e., the way in which the average velocity 
changes when a constant force is added.

We fix a large time $u$ and we define the time-averaged mobility by

$$
\mathcal{X}_{j k}(u)=\left.\frac{1}{u} \int_{0}^{u} \mathrm{~d} t \frac{\partial}{\partial h}\left\langle p_{k}(t)\right\rangle_{\mu}^{h}\right|_{h=0}
$$

starting from $\mu$ at time zero. We wish to connect this to the velocity fluctuations in the unperturbed (but driven) system:

$$
\mathcal{D}_{j k}(u)=\frac{1}{2 u}\left\langle\left[q_{j}(u)-q_{j}(0)\right]\left[q_{k}(u)-q_{k}(0)\right]\right\rangle_{\mu}=\frac{1}{2 u} \int_{0}^{u} \mathrm{~d} t \int_{0}^{u} \mathrm{~d} s\left\langle p_{j}(s) p_{k}(t)\right\rangle_{\mu}
$$

We do not need to take the limit $u \uparrow+\infty$. In equilibrium, when the Maxwell distribution is installed with $\mu$, for $F$ and $U$ equal to zero and with all temperatures equal to $\beta^{-1}$, then $\mathcal{X}_{j k}=\frac{1}{\gamma_{j}} \delta_{j, k}$ and $\mathcal{D}_{j k}=\frac{1}{\beta \gamma_{j}} \delta_{j, k}$. Indeed, in general equilibrium systems the Einstein relation $\mathcal{X}_{j k}=\beta \mathcal{D}_{j k}$ coincides with the fluctuation-dissipation relation, [3]. This is no longer true for a system out of equilibrium, see also [4]. We give the explicit modification.

We integrate the response formula (16)-(17) for $s \in[0, u]$ where $u>t$, and then integrate $t$ again between $[0, u]$ to obtain

$$
\begin{aligned}
\mathcal{X}_{j k}(u)= & \beta_{j} \mathcal{D}_{j k}(u)-\frac{1}{2 D u} \int_{0}^{u} \mathrm{~d} t \int_{0}^{u} \mathrm{~d} s\left\langle\left[F_{j}(q(s))-\frac{\partial U}{\partial q_{j}}(q(s))\right] p_{k}(t)\right\rangle_{\mu} \\
& +\frac{1}{2 D u} \int_{0}^{u} \mathrm{~d} t\left\langle\left[p_{j}(u)-p_{j}(0)\right] p_{k}(t)\right\rangle_{\mu}
\end{aligned}
$$

This relation reduces to the familiar Einstein relation if the unperturbed system is in full equilibrium. For nonequilibrium, the last term (28) is purely inertial. We can assume exponential decay in the momentum-momentum correlation, after which the integral remains bounded for arbitrary large $u$; but then (28) is arbitrary small by the prefactor $1 / u$. For the second term in (27) we can do the integral of $p_{k}(t)$ to insert $q_{k}(u)-q_{k}(0)$. The nonequilibrium Einstein relation thus essentially corrects the equilibrium relation by the correlation between the position-dependent force imposed to the particle and the total change in position.

\subsection{Coupled oscillators}

We now consider coupled one-dimensional oscillators out of equilibrium. First we consider the stationary regime (with density of states $\rho$ ) due to different temperatures $T_{i}$. For a linear chain we can take (10) and put an oscillator at each $i=1, \ldots, n$. The conservative potential $U$ is the sum $U=\sum_{i=0}^{n} \varphi\left(q_{i+1}-q_{i}\right)$ of local couplings between the oscillators $i$ and $i+1$, with $\varphi(x)=\frac{1}{2} x^{2}+\frac{1}{4} x^{4}$. Boundary conditions are imposed by keeping $q_{0}=q_{n+1}=0$. A basic perturbation is given by switching on an external field on the $j$-th particle $V(q)=-E q_{j}$. Taking the momentum $Q=p_{k}$ at site $k$ to be the observable, the variable excess in entropy flux (13) reduces, similarly to (3), to

$$
\mathcal{S}(\omega)=-\frac{E}{T_{j}} \int_{0}^{t} \mathrm{~d} s p_{j}(s) h_{s}
$$

while the frenesy equals

$$
\tau(\omega)=-\frac{E}{D} \int_{0}^{t} \mathrm{~d} s\left[\dot{p}_{j}(s)+\frac{\partial U}{\partial q_{j}}(q(s))\right] h_{s}
$$



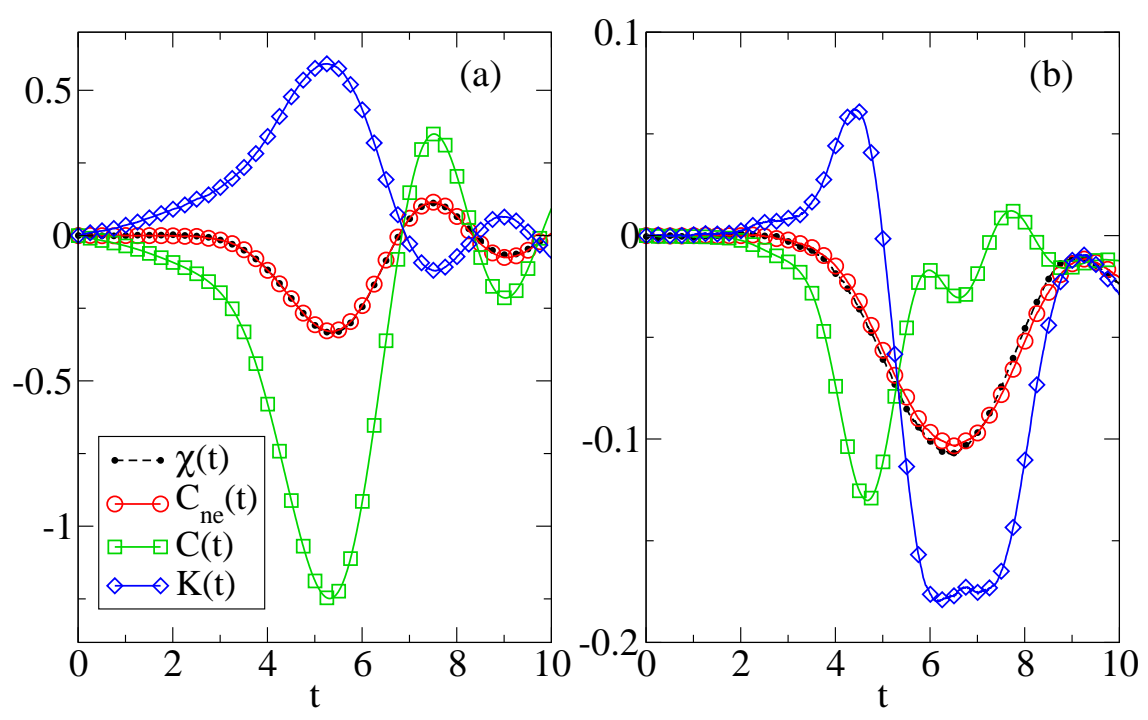

Figure 3: Visualization of the fluctuation-response relation $\chi(t)=C_{\text {ne }}(t)$, for $n=11$ coupled oscillators, with parameters, $D=0.01, h=0.03$, and (a) $T_{i}=i / 10$, (b) $T_{i}=0.2$ and transient regime as described in the text.

From (16) and (17) and quite similar to (5), we have

$$
\left.\frac{\delta}{\delta h_{s}}\left\langle p_{k}(t)\right\rangle_{\rho}^{h}\right|_{h=0}=-\frac{E}{2 T_{j}}\left\langle p_{j}(s) p_{k}(t)\right\rangle_{\rho}-\frac{E}{2 D} \frac{\mathrm{d}}{\mathrm{d} s}\left\langle p_{j}(s) p_{k}(t)\right\rangle_{\rho}-\frac{E}{2 D}\left\langle\frac{\partial U}{\partial q_{j}}(q(s)) p_{k}(t)\right\rangle_{\rho}
$$

This last relation is still valid for all times $s, t$ and is automatically equal to zero for $s>t$ (causality). Let us write $\beta_{j}=\frac{\gamma_{j}}{D}=1 / T_{j}$ and rearrange formula (29) for the situation where $s<t$ :

$$
\begin{aligned}
\left.\frac{\delta}{\delta h_{s}}\left\langle p_{k}(t)\right\rangle_{\rho}^{h}\right|_{h=0}= & -E\left(\frac{\beta_{j}+\beta_{k}}{2}\right)\left\langle p_{j}(s) p_{k}(t)\right\rangle_{\rho} \\
& -\frac{E}{2 D}\left(\left\langle\frac{\partial U}{\partial q_{j}}(q(s)) p_{k}(t)\right\rangle_{\rho}+\left\langle p_{j}(s) \frac{\partial U}{\partial q_{k}} q(t)\right\rangle_{\rho}\right)
\end{aligned}
$$

Note that the right-hand now shows a formal space-time symmetry for exchanging $j \leftrightarrow k, s \leftrightarrow t$. In equilibrium the symmetry is true on spatial level alone, $j \leftrightarrow k$, because time-symmetry is automatic. That is then an instance of Onsager reciprocity.

Choosing again constant $h=0$ for times $s>0$, in Fig. 3(a) we show the response in a system with $n=11$ oscillators, with a linear gradient of temperature $T_{i}=i / 10=1 / \beta_{i}$, perturbation applied on site $j=1$ and response tested at central site $k=6$.

We finally recall that our formulas work also for transient regimes. In Fig. 3(b) we have the response of a system with constant temperatures $T_{i}=0.2$, but where we start from a state out of equilibrium, by choosing $p_{1}(0)=10$ and the other momenta equal to zero.

\section{Conclusions}

We have shown that the nonequilibrium linear response relations for open inertial dynamics split in two terms. The first is entropic and makes the correlation of the observable at time $t>s$ with the excess 
in entropy flux at time $s$. That can involve dissipation over different reservoirs, each one with its own equilibrium temperature. The second term depends on more details of the model dynamics and we have argued it should be understood as an excess in dynamical activity or generalized escape rate. So far that activity, which we also called frenesy in linear approximation, is not understood operationally for real experiments but it is expressed as a statistical average and correlation function of theoretically known forces.

Acknowledgments: B.W. receives support from FWO, Flanders. M.B. acknowledges financial support from the University of Padua (Progetto di Ateneo n. CPDA083702) and hospitality from the Institute of Theoretical Physics at the K.U.Leuven.

\section{References}

[1] M. Baiesi, C. Maes and B. Wynants: Fluctuations and response of nonequilibrium states, Phys. Rev. Lett. 103, 010602 (2009).

[2] M. Baiesi, C. Maes and B. Wynants: Nonequilibrium Linear Response for Markov Dynamics, I: Jump Processes and Overdamped Diffusions, J. Stat. Phys., DOI 10.1007/s10955-009-9852-8 (2009).

[3] V. Balakrishnan: Elements of Nonequilibrium Statistical Mechanics, Ane Books India (2008).

[4] W. Blickle, T. Speck, C. Lutz, U. Seifert, and C. Bechinger: Einstein relation generalized to nonequilibrium, Phys. Rev. Lett 98, 210601 (2007).

[5] H. B. Callen and T. A. Welton: Irreversibility and generalized noise, Phys. Rev. 83, 34 (1951).

[6] R. Chetrite, G. Falkovich and K. Gawędzki: Fluctuation relations in simple examples of nonequilibrium steady states: J. Stat. Mech. P08005 (2008).

[7] G. Diezemann: Fluctuation-dissipation relations for Markov processes, Phys. Rev. E 72, 011104 (2005).

[8] J. R. Gomez-Solano, A. Petrosyan, S. Ciliberto, R. Chetrite, and K. Gawȩdzki: Experimental verification of a modified fluctuation-dissipation relation for a micron-sized particle in a non-equilibrium steady state, Phys. Rev. Lett. 103, 040601 (2009).

[9] T. Harada and S.-Y. Sasa: Equality connecting energy dissipation with violation of fluctuationresponse relation, Phys. Rev. Lett. 95, 130602 (2005).

[10] S. Katz, J.L. Lebowitz and H. Spohn: Phase Transitions in Stationary Non-equilibrium States of Model lattice Systems, Phys. Rev. B 28, 1655-1658 (1983).

[11] M. Krüger and M. Fuchs: Non-Equilibrium relation between mobility and diffusivity of interacting Brownian particles under shear, Progress of Theoretical Physics Supplement, arXiv:0911.1632v1 [cond-mat.soft].

[12] M. Krüger and M. Fuchs: Phys. Rev. Lett. 102, 135701 (2009).

[13] R. Kubo: The fluctuation-dissipation theorem, Rep. Prog. Phys. 29, 255-284 (1966). 
[14] J. L. Lebowitz: Stationary Nonequilibrium Gibbsian Ensembles, Physical Review 114, 1192-1202 (1959).

[15] J. L. Lebowitz and H. Spohn: A Gallavotti-Cohen type symmetry in large deviation functional for stochastic dynamics, J. Stat. Phys. 95, 333-365, 1999.

[16] E. Lippiello, F. Corberi, A. Sarracino, M. Zannetti: Nonlinear susceptibilities and the measurement of a cooperative length, Phys. Rev. B 77, 212201 (2008)

[17] E. Lippiello, F. Corberi, and M. Zannetti: Fluctuation dissipation relations far from equilibrium, J. Stat. Mech. P07002 (2007).

[18] C. Maes: Fluctuation theorem as a Gibbs property, J. Stat. Phys. 95, 367-392 (1999).

[19] C. Maes: On the origin and the use of fluctuation relations for the entropy, In: Séminaire Poincaré 2, Eds. J. Dalibard, B. Duplantier, and V. Rivasseau (Birkhäuser, Basel, 2003), pp. 29.

[20] C. Maes and K. Netočný: Time-reversal and entropy, J. Stat. Phys. 110, 269-310 (2003).

[21] C. Maes, K. Netočný, and B. Wynants: Steady state statistics of driven diffusions, Physica A 387, 2675-2689 (2008).

[22] C. Maes, K. Netočný and B. Wynants: On and beyond entropy production; the case of Markov jump processes, Markov Proc. Rel. Fields 14, 445-464 (2008).

[23] C. Maes, and B. Wynants: On a response function and its interpretation, for Markov Proc. Rel. Fields (2009).

[24] P.A.Martin: Physique statistique des processus irreversibles, Lecture Notes of the DEA de Physique Théorique, notes by F.Coppex, ENS Lyon, Fall 2001-Spring 2004.

[25] A. Puglisi, A. Baldassarri and A. Vulpiani: Violation of the Einstein relation in Granular Fluids: the role of correlations, J. Stat. Mech. P08016 (2007).

[26] A. Puglisi, D. Villamaina: Irreversible effects of memory, Europhys. Lett. 8830004 (2009).

[27] D. Ruelle: A review of linear response theory for general differentiable dynamical systems, Nonlinearity 22, 855-870 (2009).

[28] U.Seifert and T.Speck: The Fluctuation-Dissipation Theorem for Nonequilibrium Steady States: Role of Stochastic Entropy and a Classification of Variants, arXiv:0907.5478v1 [cond-mat.stat-mech].

[29] T.Speck and U.Seifert: Restoring a fluctuation-dissipation theorem in a nonequilibrium steady state, Europhys. Lett. 74, 391-396 (2006).

[30] Hal Tasaki: Two theorems that relate discrete stochastic processes to microscopic mechanics, arXiv:0706.1032 [cond-mat.stat-mech].

[31] D. Villamaina, A. Baldassarri, A. Puglisi and A. Vulpiani: The fluctuation-dissipation relation: how does one compare correlation functions and responses? J. Stat. Mech. P07024 (2009). 